\title{
Silk Patterns: Conservation and Development of Traditional Thai silk Production for Added Commercial Value in Khon Kaen Province
}

\author{
Kasinee Sawasdee ${ }^{1}$, Boonsom Yodmalee ${ }^{1} \&$ Kosit Phaengsoi ${ }^{1}$ \\ ${ }^{1}$ The Faculty of Cultural Science, Mahasarakham University, Khamriang Sub-District, Kantarawichai District, \\ Maha Sarakham Province, Thailand \\ Correspondence: Kasinee Sawasdee, The Faculty of Cultural Science, Mahasarakham University, Khamriang \\ Sub-District, Kantarawichai District, Maha Sarakham Province 44150, Thailand. E-mail: \\ ksawasdee144@hotmail.com
}

Received: June 9, 2014 Accepted: July 14, 2014 Online Published: September 22, 2014

doi:10.5539/ach.v7n1p41 URL: http://dx.doi.org/10.5539/ach.v7n1p41

\begin{abstract}
Silk patterns are examples of fine art that show the valuable culture and identity of Thai communities. This is a qualitative research and the researchers used a purposive sampling technique to identify four districts in Khon Kaen province for assessment by means of survey, observation, interview, focus group discussion and workshop. The history and development of silk patterns in Khon Kaen province occurred from a process of pattern making called mudmee. The techniques were passed from generation to generation, copying plant and animal patterns in nature. Silk patterns developed in three ways: 1) using mudmee patterns as a model; 2) imitating television, fashion magazines and other media; 3 ) following specific commission specifications of customers. Production processes are mudmee (silk blending) and tammee (silk marking), which require original wooden equipment reinforced with steel for strength and electrical motors for speed. Both simple silk fibres and factory silk fibres are used and patterns are created based on traditional designs. In order to develop silk pattern production for added commercial value, original patterns with contemporary character must be chosen and expanded as bigger and more varied products, such as handbags. New silk patterns sold in local and regional markets will boost the income of people in Khon Kaen Province.
\end{abstract}

Keywords: silk, patterns, production, product development, conservation

\section{Introduction}

Weaving reveals the inherited identity of ethnic groups. Woven products are produced in response to everyday needs and are physical representations of culture, traditions, ideas and beliefs (Suwan, 2009). In Thailand, there is archaeological evidence suggesting that white mulberry (Morus alba) has been cultivated and silkworms (Bombyx mori) bred for over three thousand years. The long history of silk weaving has allowed the development of many weaving processes, dyeing techniques and pattern designs that show different community identities, social conditions, lifestyles and traditional knowledge (Methawin, 2008).

In the past, Thai silk was not accepted by the world market and, consequently, domestic popularity suffered. This situation changed after World War II, when American businessman Jim Thomson revived the Thai silk industry and increased the reputation of Thai silk across the world (Klungpanyathai, 2009). North-eastern Thai, or Isan fabric is regarded as some of the best quality fabric in the country due to the weaving patterns and colours that differ in each locality. The most woven fabrics of Isan are khid and mudmee, while in the North almost all weavers produce cloth based on Lanna styles and in the South yok is the principal fabric. Although modern weaving is becoming homogenised, it is possible to find local products that retain their original identity (Silpakorn University, 2001).

Fibre production is not easy. In addition to loom weaving of natural coloured fabric, weavers incorporate their own pattern variations. The patterns are often influenced by everyday objects, including key patterns (pra jae), hook patterns $(k h o)$ and pigtail patterns (kha-pia). Other patterns include the tum pattern, based on fishing equipment; flower or fruit based ba and krabok patterns; floral dok sarn, dok jik, dok yung, dok kaew and dok pi kul patterns; phong nam sponge patterns; and kho kra jorn patterns, replicating earrings. Aside from everyday objects, patterns are also derived from beliefs and imagination, such as the patterns of nak kho, nak hua chor and nak khon tee (Office of Archaeology and the National Museum, 1997). Silk patterns have been inherited from 
generation to generation and reveal the values, lifestyles, visions, traditions and culture of women in Isan, who were the primary weavers. Isan people consider silk a valuable commodity and are proud of their ancestral weaving traditions (Phojanee, 2000).

Khon Kaen province is the most important silk production area in modern Isan and production groups in the region emphasise silk production to add commercial value to community enterprises. There are many important and outstanding silk production businesses. Chonnabot District is the most famous district for silk production and particularly mudmee silk (Nartnapang, 2005). Mudmee silk patterns in Khon Kaen province have developed so fast and so extensively that outsiders are ignorant of the original patterns, weavers imitate eye-catching styles and the silk products have lost their traditional local identity. New products combine traditional knowledge with modern technology and the designs are pre-produced using graph paper and computer programs. Problems occur when the older generations, who are the sole source of information regarding traditional patterns, cannot blend either old or new patterns because they do not have the skill to use modern machinery.

For this reason and given the traditional importance and value of Isan silk weaving, the research team wished to analyse conservation and development of traditional Thai silk production for added commercial value in Khon Kaen Province. The objective was to conserve and develop original silk patterns, integrating new concepts in silk pattern design and production to meet local requirements and appeal to consumers. It is hoped that the knowledge gathered from this research can be applied to promote community economy and job creation in North-eastern Thailand.

\section{Methodology}

This is a qualitative research with two main aims: 1) To study the history and development of Thai silk patterns in Khon Kaen province; 2) To study the conservation and development of Thai silk production for added commercial value in Khon Kaen province. The researchers used a purposive sampling technique to identify six mudmee silk production groups in four districts of Khon Kaen province for analysis. The research area has a fifty-year history of commercial silk production integrating traditional knowledge inherited from ancestral communities. The key informants were academics, teachers, local community members and production group leaders. The casual informants were manufacturers in the four districts. The general informants were traders and consumers. Tools used for data collection were survey, participant and non-participant observation, structured and unstructured interview, focus group discussion and workshop. The data collected during field study and document research was organized according to the two aims of the investigation. Data was validated using a triangulation method, as documented by Supang Chantavanit (2002). The research data was analyzed using analytic induction, typological analysis and constant comparison. The study began in March 2012 and ended in June 2013.

\section{Results}

The first mudmee silk production group was formed in 1984. In the past, two silk patterns were produced in Khon Kaen Province, mak jub (Figure 1) and kho lho (Figure 2). Mak jub was the archetype that all mudmee silk leaders in Khon Kaen Province learned and inherited. Production knowledge was especially important in Chonnabot District, where makjub was first conceived (Thongsook, 2012).

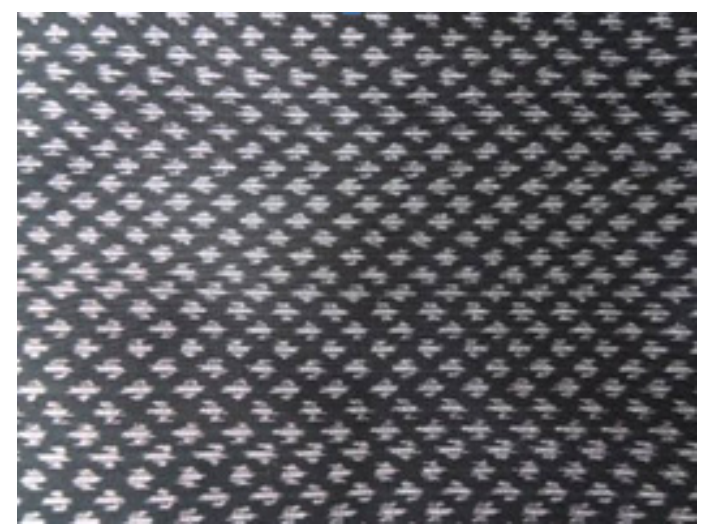

Figure 1 . The identity of makjub patterns 


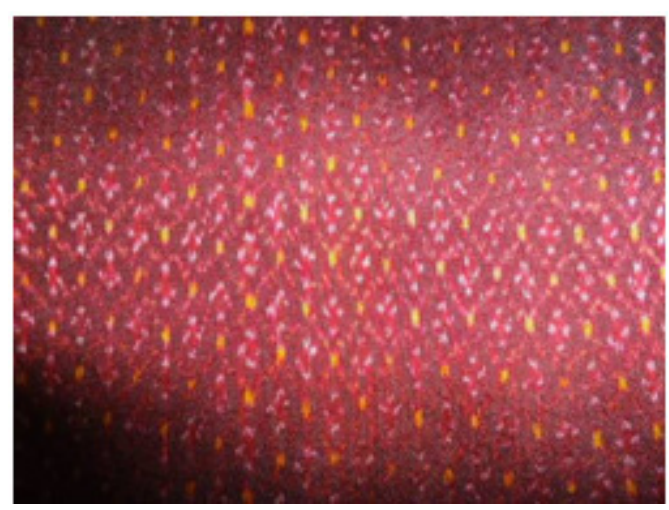

Figure 2. The identity of kho lho patterns

At present, various patterns are produced in Khon Kaen Province. Most of these designs are imitations from elsewhere and originate in external consumer fashion and from the media. Designs are generally produced in the community and made repeatedly. Occasionally the patterns are named. These named designs give an identity to the founding production group and are transformed to create male and female clothes (Supanee, 2011). Local pattern identities are:

- Phong nam hua phay (Figure 3) of Ban Hua Phay in Po Daeng Sub-district, Chonnabot District.

- Jee phetch (Figure 4) of Ban Nong Krong Kaew in Chonnabot Sub-district, Chonnabot District.

- Animal patterns such as kai, or chicken pattern (Figure 5) and five-heddle spot pattern nop pa kaow (Figure 6) of Ban Chonnabot in Si Bun Rueang Sub-district, Chonnabot District.

- Nong ya plong (Figure 7) of Ban Nong Ya Plong in Phon Phek Sub-district, Mancha Khiri District.

- Buy sri pha ya nak (Figure 8) of Ban Kham Wari in Mueang Phia Sub-district, Ban Phai District.

- Kho sam sib jed (Figure 9) of Ban Chad in Kham Pom Sub-district, Phra Yuen District.

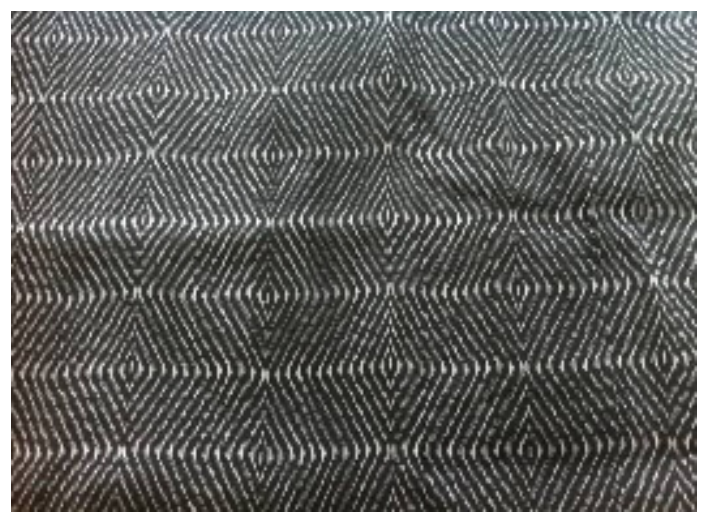

Figure 3. The identity of phong nam hua phay patterns 


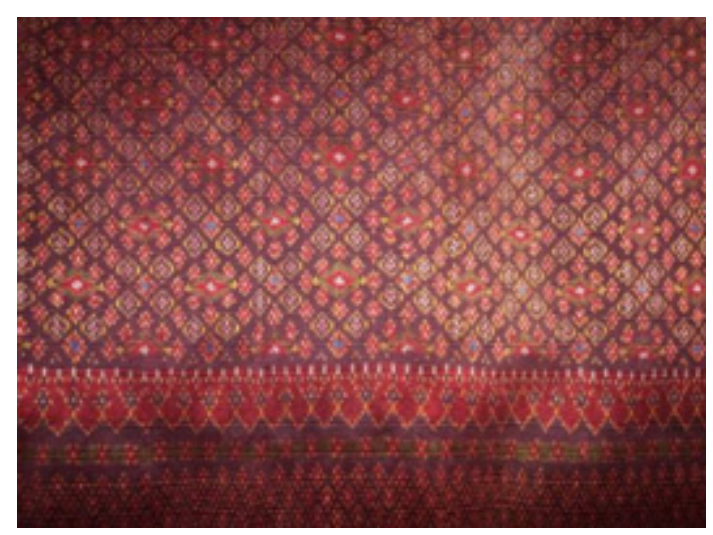

Figure 4 . The identity of jee phetch patterns

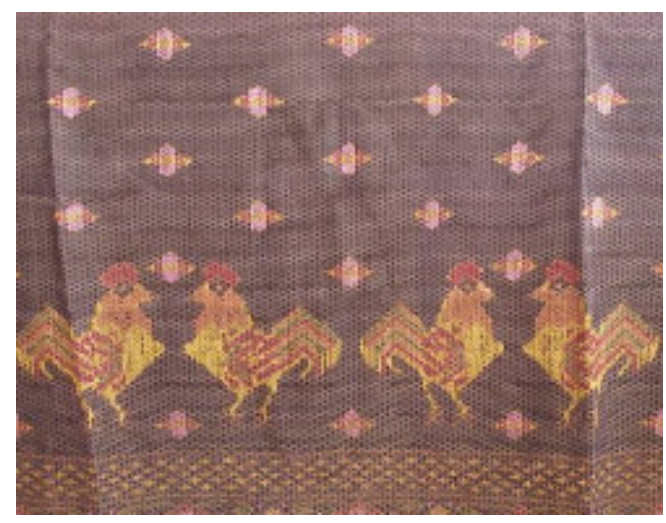

Figure 5. The identity of animal patterns (chicken)

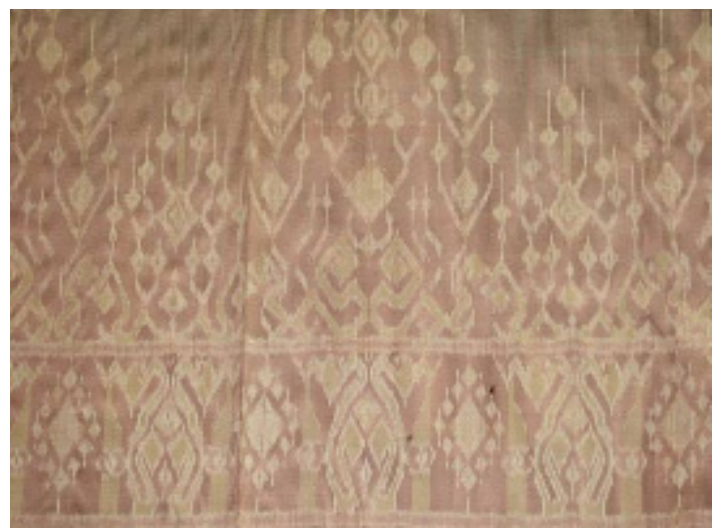

Figure 6. The identity of nop pa kaow patterns (five heddles) 


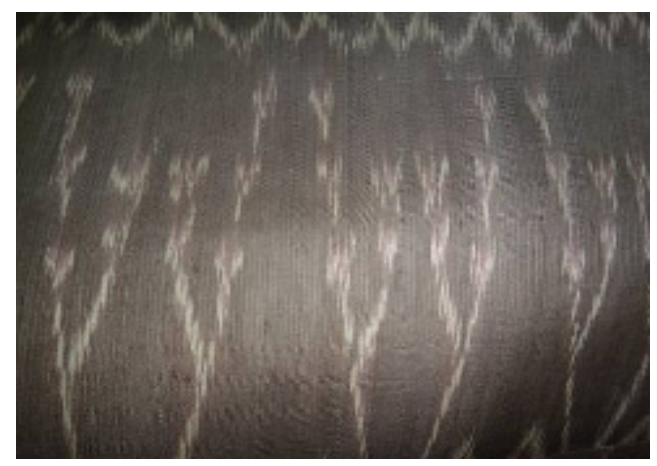

Figure 7. The identity of nong ya plong patterns

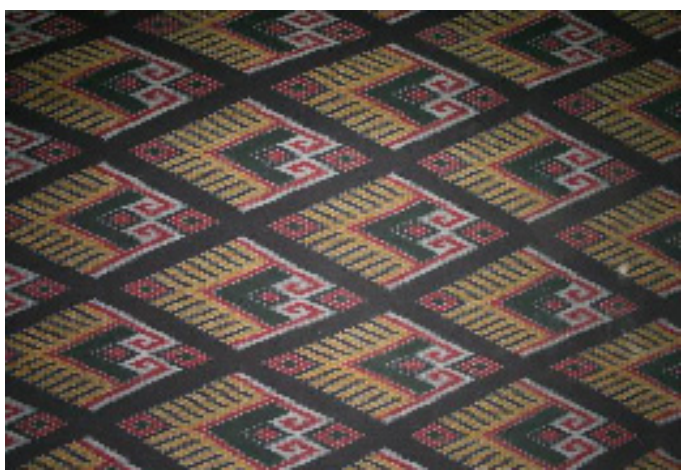

Figure 8. The identity of buy sri pha ya nak patterns

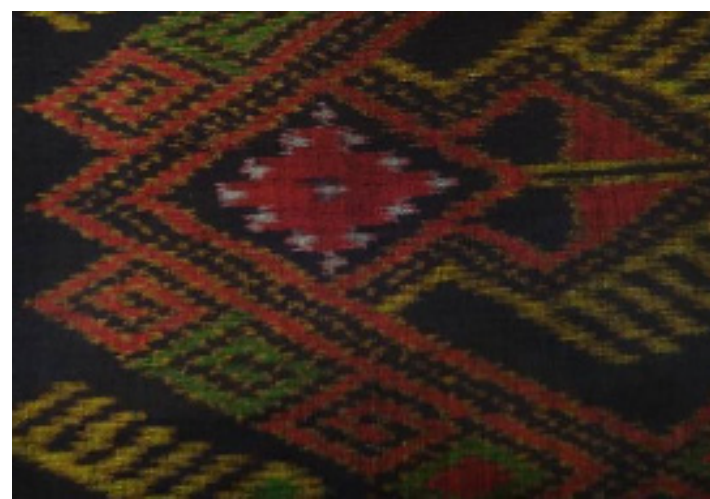

Figure 9. The identity of kho sam sib jed patterns

Production groups in every district use original patterns as models to design and develop pattern varieties. The method of pattern design is remembered by experts, who blend the patterns to create new versions. Production groups in every district use graph paper to make designs before blending patterns. Ban Chad silk production group in Phra Yuen District is the only group not to continue the development stage on computer. Computer designs help modernise creations and the electronic samples can be used in communication with consumers. Two groups, Ban Hua Phay silk production group in Chonnabot District and Ban Nong Ya Plong silk production group in Mancha Khiri District consult consumers using computer designs. Production groups in every district design patterns to consumer specifications, which is a way of creating valuable local identity and competing in the marketplace.

\subsection{Development of Equipment and Processes in Mudmee Silk Pattern Production in Khon Kaen Province}

The two traditional processes of mudmee and tammee have been conserved in Khon Kaen Province. Nevertheless, there have been innovations in the silk production process that have reduced production times, 
expenses, wages and operation steps. There are also new colour variations thanks to knowledge developments in primary colours and colour mixing. Traditional weaving equipment consists of a reel for spinning the silk (auk), a wooden frame (hong khon mee) for silk spun by hand, a blade for fixing the silk, a wooden wheel and a wooden loom. The traditional equipment has been developed by reinforcing the wooden frame with steel and adding a motor for spinning. The production process remains the same as the past. The weft fibres are prepared for pattern blending. The silk is then purified and spun. The weft fibres are arranged by length along the reed of the loom. The specifications for the weft are set (khon lam mee) for making specific patterns. The mudmee frame is then filled and the silk is blended with other dyed fibres to create patterns and spun. The blended fibres are finally woven into a fabric using a loom (Songkram N, personal communication, 2011).

\subsection{Conservation of Silk Pattern Production for Added Commercial Value}

Traditional patterns are ultimately conserved in the locality because the community has a traditional knowledge base and does not actively learn new techniques. There are new design innovations of silk patterns that have never been produced in the community but these do not cause the original patterns to disappear. Instead, new developments make the community realize the value and worth of original patterns. Moreover, commercial value is added because, by producing original patterns in which the community has knowledge and skill, they reduce production time. Nonetheless, modern patterns are demanded by consumers and can sell at a high price when compared to patterns of silk product that have been produced for a long time. Therefore, active conservation of original patterns with expansion to bigger and more varied styles is the way to add commercial value to the silk of Khon Kaen Province.

There are two types of pattern development: completely new designs and applied traditional patterns. Four traditional patterns that are commonly adapted are mak jub yai, mak bok yai, mak jub yai pra yook and dok kaew yai. These patterns have proven to be good for sale and mass production. Silk patterns are usually made by coupling colours. White or cream will often be used as the main colour of the silk fabric and blended with only one additional colour. This is to reduce dyeing costs and increase production speed. New products are then created by transforming the silk patterns into products, such as mak jub yai and mak jub pra yook handbags with wooden handles, mak bok yai silk patterned handbags with rattan palm and wooden handles, mak jub yai and mak jub yai pra yook box handbags, mak jub silk patterned pyramidal handbags and small bags from silk off cuts. Silk pattern products are easy to sell and increase profits because previously only sheet fabrics were sold. The products allow for market expansion and attract a new class of consumer, different age groups and occupations.

\section{Discussion}

From study of six mudmee silk production groups in four districts Khon Kaen Province, it is not clear exactly when mudmee silk weaving first occurred but there is evidence to suggest it has been learnt and inherited for over 3000 years. The original patterns are imitated from nature and imagined from religious beliefs. These ideas correspond to the research of Surachaipataradit Lordkamwattana, which concerned local knowledge of Tai Yuan native fabric weavers in Nakhon Ratchasima Province. The results show that, although there was a variety of fabric patterns, most were derived from nature and the village environment (Lordkamwattana, 2009).

At present, a variety of silk patterns are produced in each locality and each region has its own fabric identity. This corresponds to the theory of symbolic interactionism, which focuses on the idea of understanding symbols and processes as interpretations of human interaction. Primary symbols drive human understanding, meaning and interaction, so each group will produce silk patterns that reflect its own local identity (Chantachon, 2006). This idea agrees with the research of Lakana Thanawanakit, which studied consumer satisfaction with jok fabric products in Ratchaburi Province. Thanawanakit found that patterns appearing on fabric will show the identity, culture and lifestyle of people who weave. Over time, economic status and society will change the perspectives of the weavers and cause patterns to evolve (Thanawanakit, 1997).

Mudmee silk production uses the same tools and processes as the past but steel has been added to reduce maintenance costs and motors have been applied to decrease production time. Udom Choeikeewong argued that these innovations are necessary developments because they improve, restore and format culture so that it does not disappear from one generation to the next. As a result, cultural heritage and the daily life of local people both benefit (Choeikeewong, 2005).

There are adaptations to four traditional silk patterns: mak jub yai, mak bok yai, mak jub yai pra yook and dok kaew yai. These silks are transformed into a variety of products that have not previously appeared in the local markets and can increase the value of the silk. New silk pattern products retain their quality but profits have increased because overhead capital and labour time have decreased, goods are easier to sell, prices are higher and 
they can compete in the market. These developments were also noticed by Niyom Wongpongkham in a study of traditional Isan knowledge for the production of bronze alloy products. While products retain the original concepts, local knowledge has been integrated with new ideas, especially material, production process and product form development. This adds value to the traditional handicrafts as it meets the requirements of the community and consumers (Wongpongkham, 2008).

The researchers make five suggestions for further research to improve mudmee silk patterns in Khon Kaen:

- Appropriate colour combinations should be determined.

- Factors affecting success and failure of pattern conservation and development must be analysed.

- Cultural environment conditions that affect conservation and development of silk pattern production should be identified.

- Silk pattern products should be formatted to aid marketing efficiency.

- Financial analysis and specifications of silk pattern products should be conducted to determine appropriate pricing scales for silk products.

\section{Conclusion}

The history and development of silk patterns in Khon Kaen province occurred from a process of pattern making called mudmee. The techniques were passed from generation to generation, copying plant and animal patterns in nature. Silk patterns developed in three ways: 1) using mudmee patterns as a model; 2) imitating television, fashion magazines and other media; 3 ) following specific commission specifications of customers. Production processes are mudmee (silk blending) and tammee (silk marking), which require original wooden equipment reinforced with steel for strength and electrical motors for speed. Both simple silk fibres and factory silk fibres are used and patterns are created based on traditional designs. In order to develop silk pattern production for added commercial value, original patterns with contemporary character must be chosen and expanded as bigger and more varied products, such as handbags. New silk patterns sold in local and regional markets will boost the income of people in Khon Kaen Province. Successful conservation and development of silk pattern production in Khon Kaen Province adds commercial value to community handicrafts. If the local producers accept new ideas that integrate traditional knowledge, their work with cultural capital will add to the market power of local silk products.

\section{References}

Chantachon S. (2006). Qualitative research [in Thai]. Kalasin: Prasan Publishing.

Chantavanit, S. (2002). Qualitative research method [in Thai]. Bangkok: Chulalongkorn University Press.

Choeikeewong U. (2005). Art and Thai culture [in Thai]. Bangkok: Sangdow.

Klungpanyathai. (2009). Thai textiles. Retrieved July 17, 2011, from http://www.panyathai.or.th

Lordkamwattana S. (2009). Knowledge of Thai Yuan native weaving: Method of business management of community, Si Kuew District, Nakhon Ratchasima province [in Thai]. PhD thesis, Mahasarakham University, Thailand.

Methawin S. (2008). Royal grace of Queen Sirikit with native weaving [in Thai]. Thai Culture, 47(9).

Nartnapang Y. (2005). Diagram of the future mudmee industry, Chonnabot District, Khon Kaen Province [in Thai]. PhD thesis. Khon Kaen University, Thailand.

Office of Archaeology and the National Museum. (1997), Native Isan fabric [in Thai]. Bangkok: Prescale.

Phojanee T. (2000). Silk pattern research project: Knowledge integration and strengthening in community for developing the quality of life of children and youth: A case study of the royal idea project of Princess Sirindhorn in North-eastern Thailand [in Thai]. PhD thesis, Mahasarakham University, Thailand.

Silpakorn University. (2001). Native textiles and survey of nationwide manufacturers [in Thai]. Bangkok: Silpakorn University Press.

Suwan, P. (2009). Culture and religion [in Thai], Bangkok: Ramkhamhang University.

Thanawanakit, L. (1997). Consumer satisfaction with jok fabric products in Ratchaburi [in Thai]. PhD thesis. Kasetsart University, Bangkok.

Wongpongkham, N. (n. d.). Traditional Isan knowledge for production of bronze alloy products for developing commercial value [in Thai]. $\mathrm{PhD}$ thesis, Mahasarakham University, Thailand. 


\section{Copyrights}

Copyright for this article is retained by the author(s), with first publication rights granted to the journal.

This is an open-access article distributed under the terms and conditions of the Creative Commons Attribution license (http://creativecommons.org/licenses/by/3.0/). 